\title{
Laparoscopic Surgery of Deferential Reflux in Pediatric and Adolescent Varicocele
}

\author{
Marcello Cimador, MD, Maria R. Di Pace, MD, Maria Sergio, MD, Pieralba Catalano, MD, \\ Marco Castagnetti, MD, and Enrico De Grazia, MD
}

\begin{abstract}
Background: This study aimed to assess whether deferential reflux in pediatric and adolescent varicocele can be successfully treated laparoscopically.

Materials and Methods: Since 2001 at our institution, 148 boys were evaluated for a left varicocele. Preoperatively, all the patients underwent ultrasound scan assessment of testicular volume and color-Doppler US (CDUS) to rule out reflux into the internal spermatic vein (ISV), deferential vein, or cremasteric vein. Boys with ISV reflux were treated by laparoscopic transperitoneal Palomo; boys with isolated deferential reflux or associated to ISV reflux were laparoscopically managed adding to the former procedure, coagulation or clipping of refluxing deferential veins.

Results: Reflux in both the ISV and the deferential vein was observed in 21 (14.1\%) out 148 boys with varicocele. Only one case $(0.6 \%)$ of varicocele was caused by an isolated deferential reflux. No reflux in the cremasteric vein was observed. After a median follow up period of 2 years (range, 6 months -5 years), none of our patients with deferential reflux experienced varicocele recurrence either clinically or according to CDUS scanning. No testicular atrophy was observed.

Conclusion: Our diagnostic approach is a rigorous standard for identifying all the venous systems concurring with the varicocele. Our proposed technique with laparoscopic interruption or coagulation of deferential veins when proved by CDUS to be refluxing may allow successful treatment for most varicoceles. This method allows reduction in recurrence of varicocele due to a missed deferential reflux.
\end{abstract}

\section{Introduction}

$\mathbf{T}$ HE IDEAL METHOD for varicocele treatment is still controversial. Actually, varicocele repair may be accomplished by open surgery, laparoscopy, or interventional radiology. ${ }^{1-2}$ Besides, treatment may be performed at different levels, including retroperitoneal, inguinal, and subinguinal. All these methods, however, have been reported to be associated with some risk of varicocele persistence/recurrence due to collateral refluxing veins not interrupted at surgery.

Indeed, in some $75 \%$ of cases, the varicocele is caused by a reflux in a single internal spermatic vein (ISV) ${ }^{3}$ and can be treated successfully by any approach. In the remaining cases, instead, multiple refluxing venous systems may be present.

Starting from the venographic studies by Coolsaet, ${ }^{3}$ who classified varicocele in three different types, and the additional studies by Franco et al., who questioned the existence of a cremasteric reflux, ${ }^{4}$ we have developed a preoperative color-
Doppler ultrasound (CDUS) technique for the preoperative assessment of the varicocele pathophysiology. ${ }^{5}$ This method potentially allows for a preoperative noninvasive identification of the refluxing veins subtending the varicocele, and a differentiation of those actually refluxing from those dilated due to overflow. ${ }^{5-7}$ Using this tool, we have detected some patients in whom a reflux in the deferential veins was associated to the ISV reflux, and even some rare cases in whom a deferential reflux was the only cause of the varicocele. Herein we detailed our experience with the CDUS diagnosis and the laparoscopic treatment of cases of pediatric and adolescent varicocele with evidence of deferential reflux.

\section{Materials and Methods}

From January 2001 to April 2008, 148 patients with a median age of 12.4 years (range, $7.1-16$ years) were admitted at our institution for the presence of a left varicocele. All varic-

Pediatric Surgical Unit, Department of Mother and Child Care, Università di Palermo, Palermo, Italy. 
oceles were graded by scrotal physical examination according to Dubin and Amelar ${ }^{8}$ in the supine and upright positions. Patients underwent surgical treatment if presenting with a grade 3 varicocele or a grade 2 varicocele, this latter if associated with hypotrophy of the left testicle, pain or scrotal discomfort. Preoperatively, all the patients underwent ultrasound scan assessment performed using a linear multifrequency 7 to $13 \mathrm{MHz}$ transducer connected to an Aloka Prosound SSV500, (Tokyo, Japan). For CDUS pulse repetition, the frequency was set at $1.5 \mathrm{kHz}$. Using ultrasound, testicular volume was calculated in milliliters with the formula $\mathrm{V}=0.71$ LDW of the testicular ellipsoid. ${ }^{9}$ Left scrotal, inguinal and pelvic venous networks were investigated as previously described. ${ }^{5}$ Patients presenting at surgery with dilated deferential veins proved by preoperative CDUS to be refluxing were treated by a Palomo procedure, combined with coagulation or clipping and division of deferential vein(s) as follows. A new setup of trocar position was adopted in these cases (Fig. 1). Optical was placed in right iliac fossa and two operative accesses were opened on midline, at umbilicus and hypogastrium. The retroperitoneal window, previously opened on spermatic vessels for carrying out the Palomo procedure, was widened toward the internal inguinal ring. The deferential vein(s) running as satellite(s) of the vas were gently dissected off the deferential artery, which was spared because it could remain the almost unique blood supply of the testicle. The deferential vein then was coagulated with bipolar diathermy, or clamped by clips and divided (Fig. 2). Sometimes it was easier to approach the deferential vein in the deep pelvis just beneath the iliac vessels. In these cases, a second retroperitoneal window was required. Clinical and CDUS follow-up were performed 1, 3, 6 , and 12 months postoperatively and then every year. Recurrence or persistence of the varicocele, the presence of a hydrocele, and testicular volume were evaluated. Data are reported as median and range.

\section{Results}

According to the findings, 112 boys $(75.6 \%)$ had a grade 3 and $36(24.3 \%)$ a grade 2 varicocele. A total of 16 boys with

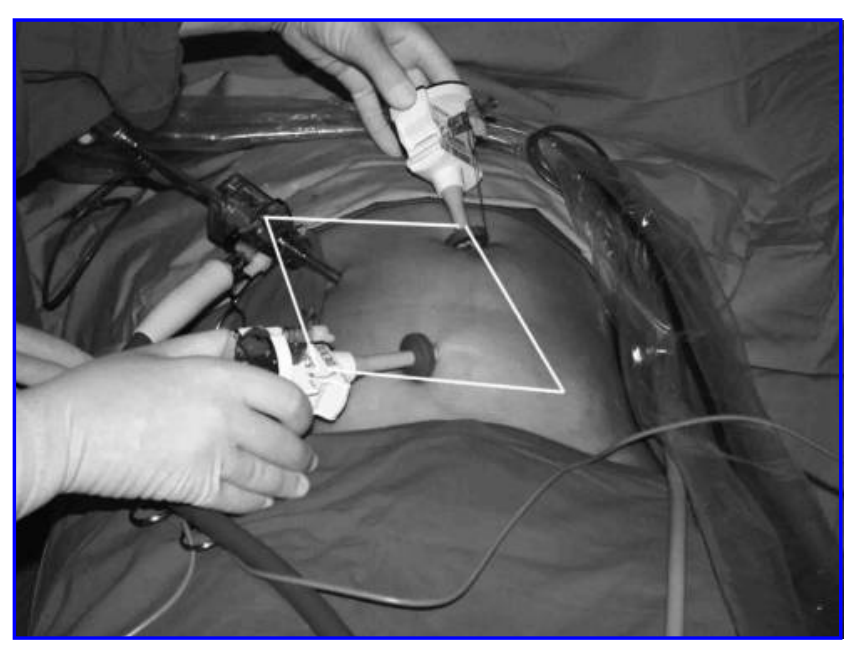

FIG. 1. Trocars setup approaching deferential reflux. Optical is inserted in right iliac fossa, whereas two operative ports are on the midline. a grade 2 varicocele reported left scrotal discomfort or pain, whereas the remaining boys had testicular asymmetry. In 126 cases $(85.1 \%)$, CDUS showed reflux in the ISV only, whereas a reflux in both the ISV and the deferential vein was observed in the remaining 21 cases (14.1\%). Only one case $(0.6 \%)$ of varicocele was caused by an isolated deferential reflux. No refluxing cremasteric vein was observed.

Based on these findings, in 21 boys with associated ISVdeferential reflux and in one boy with isolated deferential reflux, transperitoneal laparoscopic Palomo procedure was performed, adding coagulation or clipping and section of the deferential vein. In two cases, we approached the deferential vein in the deep pelvis because of a more evident dilation of the vein just underneath the left iliac vessels.

After a median follow up period of 2 years (range, 6 months -5 years), none of our patients with deferential reflux experienced varicocele recurrence either clinically or according to CDUS scanning. No case of testicular atrophy was observed. In $2(9 \%)$ out 22 patients a mild postoperative hydrocele was observed at 3 months follow-up. Both hydroceles resolved spontaneously, within 1 year. In one out two patients approached in the deep pelvis, a temporary paresthesia and a mild pain of the left thigh was recorded. These symptoms appeared immediately after surgery and spontaneously resolved within two weeks without any disability.

\section{Discussion}

Varicocele can be approached by different therapeutic strategies: percutaneous embolization or sclerosis; open surgery interruption of the spermatic vein at different levels; microsurgical venous bypass. Nevertheless no single approach can be adopted as the best. ${ }^{10-11}$ Laparoscopic or retroperitoneoscopic treatment of varicocele has recently been advocated as a simple, safe and effective technique, and it is comparable with other procedures in terms of recurrence and complication rates. ${ }^{11}$ However, a review of published reports shows that little attention has so far been paid to the different types of venous reflux (renal-internal spermatic and/or iliac-deferential reflux) in selecting the best surgical procedure. Starting from our previous studies, in our opinion a correct approach for treating varicocele should be only based on understanding of the pathophysiology. Otherwise, surgical technique should imply the mandatory interruption of all venous networks potentially causing reflux.

In our experience, preoperative CDUS study, aiming to distinguish different types of varicoceles has been considered the key-point of surgical success rate. The exact knowledge of venous refluxes involved in varicocele gave us the chance for a selected treatment in all observed cases. CDUS was an effective, noninvasive and reliable tool for investigating varicocele in all ages.

The deferential vein, which is a satellite of the vas deferens, is not detectable on CDUS in healthy boys, either at rest or under a Valsalva maneuver. It only becomes visible if dilated and refluxing. To detect such a change easily, the left external iliac artery can serve as a landmark, as the deferential vein runs over it and arches downwards. The latter can be easily identified as an arch over the external iliac vessels running from the internal inguinal orifice down the pelvis and joining the vesical vein. CDUS provides a clear 

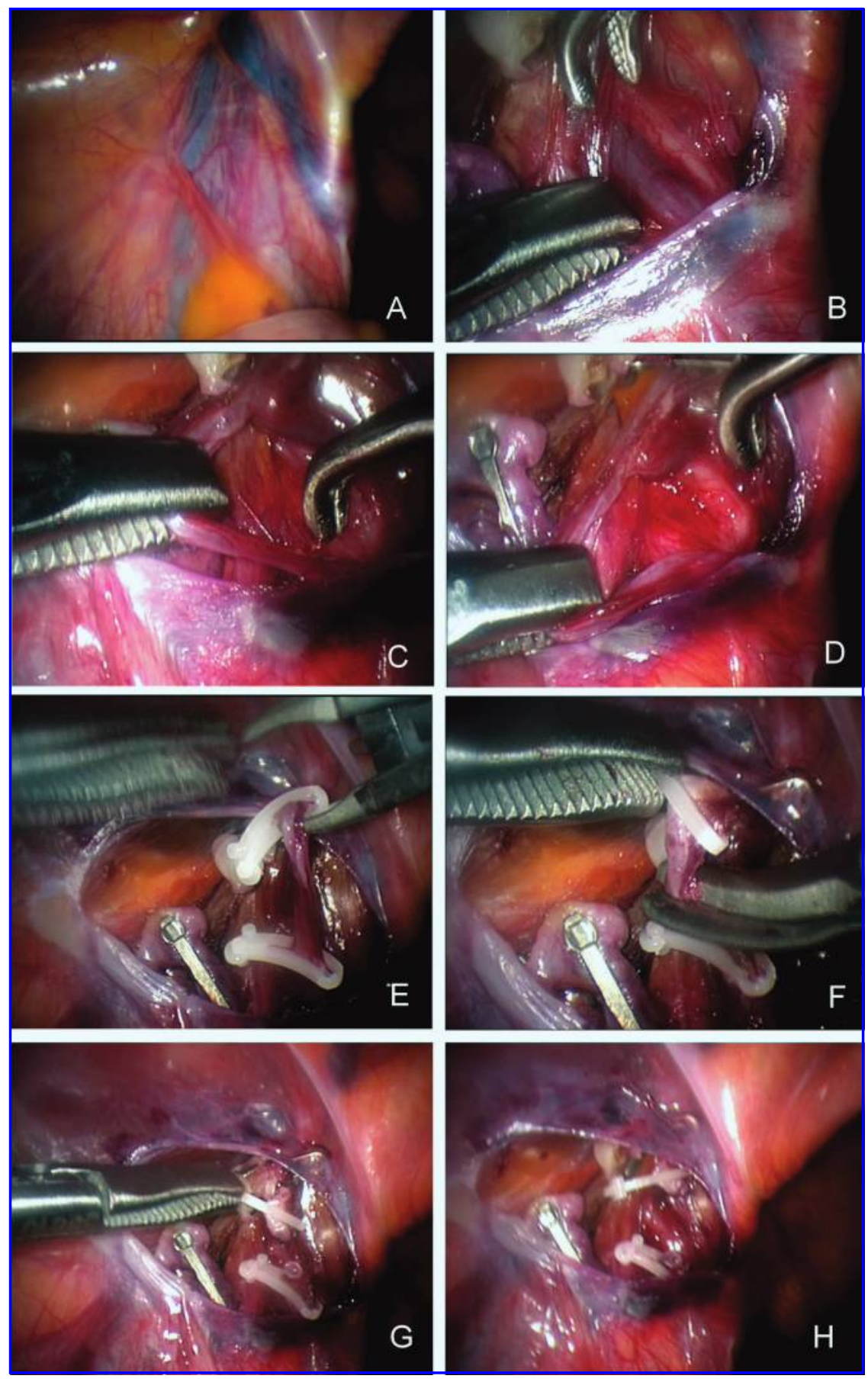

FIG. 2. Laparoscopic approach to deferential vein. (A-B) Dilated deferential vein easily detectable on laparoscopic view. (C-D) The deferential vein is picked-up far from the vas. (E-H) Division of vein after clipping.

demonstration of a deferential reflux, which can be treated by laparoscopic clipping or coagulation of deferential vein(s).

The clinical relevance of deferential reflux, its role on pathogenesis of varicocele and on ultimate adult fertility, is questionable at present. Perhaps a controlled trial may properly address this issue. For ethical reasons, we do not have a control group of untreated deferential refluxes, but involvement of deferential reflux in recurrences of varicocele has been previously demonstrated by venography. ${ }^{11,13}$
In this scenario, Dudai et al. ${ }^{12}$ suggested that should be systematically interrupted both the ISV and the inferior epigastric vessels. This procedure, however, causes unnecessary division of many non-refluxing veins and can address only a possible cremasteric reflux, whereas CDUS has allowed us to identify the refluxing venous systems preoperatively and hence to select the surgical treatment accordingly. On the basis of CDUS findings, in a previous study, we proposed to restrict open microsurgical subinguinal varicocelectomy to cases with reflux in multiple systems and to treat varicoce- 
les due to a reflux in the ISV only by a laparoscopic Palomo procedure. Doing so, we did not observe any recurrence in 42 treated cases. ${ }^{5}$ In this study, we tested the possibility of extending the laparoscopic treatment also to patients with multiple refluxing venous systems. Indeed, facing a refluxing deferential vein, we managed to dissect, coagulate, and divide this vein laparoscopically near the vas deferens. Extreme care must be paid during dissection to avoid injuries to the surrounding vessels, vas deference, and deferential artery, which in some cases could become the almost unique blood supply to the testicle. To date, however, we have not experienced any case of testicular atrophy. Interruption of the epigastric vessels could be added to the procedure for treatment of a cremasteric reflux if present. In the current series of 22 cases of deferential reflux, there was no recurrence. Furthermore, for a better approach to deferential reflux we have changed our trocars setup. Optical trocar has been moved from the umbilicus to right iliac fossa and operative trocars on the midline, in the umbilicus and hypogastrium. This different approach gave us the best view and manipulation of the deferential veins running in the deep pelvis. In few cases, we chose to manage the deferential vein in the deep pelvis, but a second retroperitoneal window was required. In these cases it is mandatory to restrict the use of electric cautery, since it may cause an injury to the genitofemoral nerve leading to post-operative sensory deficit, as observed in one case in our series. The described technique can clearly address only varicoceles due to reflux in the ISV, deferential, or cremasteric veins. Cases with other types of pelvic refluxing veins are missed. Nevertheless, the incidence of these rare refluxing vessels is very low, ${ }^{13-14}$ and their actual role in causing a clinically relevant varicocele is questionable. The proposed procedure also showed a low rate of hydroceles ( $9 \%$ ) for a nonartery-sparing technique if compared to other studies. ${ }^{11}$ However, in this series hydroceles resolved spontaneously within one year follow-up.

\section{Conclusions}

In conclusion, our diagnostic approach is a rigorous standard for identifying all the venous systems concurring with the varicocele. Our proposed technique with laparoscopic interruption or coagulation of deferential veins when proved by CDUS to be refluxing may allow successful treatment for most varicoceles. In experienced hands, it is a safe procedure and no testicular atrophy has been observed in the present study. This method dramatically reduces recurrence of varicocele due to a missed deferential reflux.

\section{Disclosure Statement}

No competing financial interests exist.

\section{References}

1. Skoog SJ, Roberts KP, Goldstein M, Pryor JL The adolescent varicocele: what's new with an old problem in young patients? Pediatrics 1996;100:112-122.

2. Diamond DA Adolescent varicocele: emerging understanding. BJU Int 2003;92:48-51.

3. Coolsaet $\mathrm{BL}$. The varicocele syndrome: venography determining the optimal level for surgical management. I Urol 1980;124:833-839.

4. Franco G, Iori F, de Dominicis C, Dal Forno S, et al. Challenging the role of cremasteric reflux in the pathogenesis of varicocele using a new venographic approach. I Urol 1999;161:117-121.

5. Cimador M, Di Pace MR, Peritore M, et al. The role of colordoppler ultrasound in determining the proper surgical approach to the management of varicocele in children and adolescents. BJU Int 2006;97:1291-1297.

6. Niedzielski J, Paduch D, Raczynsji P. Assessment of adolescent varicocele. Pediatr Surg Int 1997;12:410-413.

7. Nagar H, Mabjeesh NJ. Decision making in pediatric varicocele surgery: use of color Doppler ultrasound. Pediatr Surg Int 2000;15:75-76.

8. Dubin L, Amelar RD Varicocele size and results of varicocelectomy in selected subfertile men with varicocele. Fertil Steril 1970;21:606-609.

9. Sakamoto H, Saito K, Oohta M et al., Testicular volume measurement: comparison of ultrasonography, orchidometry, and water displacement, Urology 2007;69:152-157.

10. Kass EJ. Editorial: adolescent varicocele. I Urol 1995; 154:1483.

11. Esposito C, Monguzzi GL, Gonzales-Sabin MA et al.Laparoscopic treatment of pediatric varicocele: a multicenter study of the Italian Society of Videosurgery in infancy. J Urol 2000;163:1944-1946.

12. Dudai M, Sayfan J, Mesholam J, Sperber Y . Laparoscopic simultaneous ligation of internal and external spermatic veins for varicocele. J Urol 1995;153:704-705.

13. Niedzielski J, Paduch DA Recurrence of varicocele after high retroperitoneal repair: implications of intraoperative venography. J Urol 2001;165:927-929.

14. Campobasso P Blue venography in adolescent varicocelectomy: a modified surgical approach. J Pediatr Surg 1997; 32:1298-1301.

Address reprint requests to: Marcello Cimador, MD

Dipartimento Universitario Materno-Infantile Università di Palermo

Via Cardinale Rampolla, 1 90142 Palermo Italy

E-mail: mcimador@unipa.it 